\title{
Sigmoidal Chemorheological Models of Chip- Underfill Materials Offer Alternative Predictions of Combined Cure and Flow
}

\author{
Brian J. Love,* Fabien Teyssandier, Yang Y. Sun, Ching P. Wong
}

Prior rheology results on chip-underfill epoxy resins have been re-analyzed by a sigmoidal model that contains three variable physical parameters, including the terminal cured viscosity of the gel, an induction or dwell time and a time factor associated with the speed of conversion as viscosity undergoes large dynamic changes during rapid crosslinking. The analyses were conducted with resins that were originally cured between 150 and $180^{\circ} \mathrm{C}$ and show obvious non-linearity, even on a semi-log plot of dynamic viscosity. The sigmoidal models more accurately represent a wider range of dynamic viscosity than power-law-based rheological models, which are both more common and more generally accepted for practical application. If total flow is the critical design parameter in terms of chip underfill, perhaps these alternative sigmoidal models need to be more thoroughly evaluated to gauge their practical use and validity.

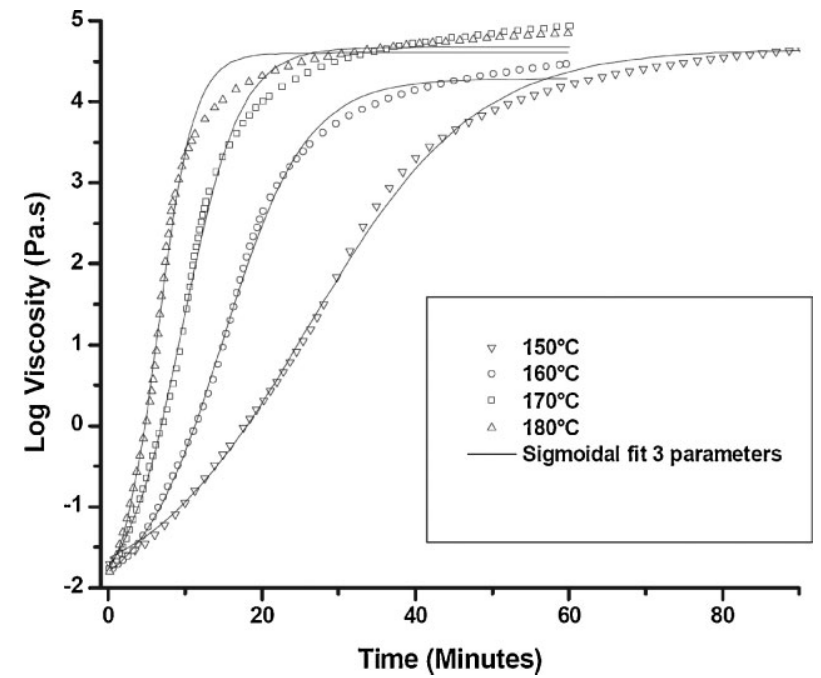

B. J. Love, F. Teyssandier

Department of Materials Science and Engineering,

University of Michigan, Ann Arbor, Ml 48109

E-mail: bjlove@umich.edu

B. J. Love

Department of Biomedical Engineering, University of Michigan, Ann Arbor, MI 48109

B. J. Love

Department of Biologic and Materials Sciences (Dentistry),

University of Michigan, Ann Arbor, MI 48109

Y. Y. Sun, C. P. Wong

School of Materials Science and Engineering, Georgia Institute of

Technology, Atlanta, GA 30332

\section{Introduction}

There is significant interest in establishing molecular parameters linked with the gel point: ${ }^{[1]}$ the induction time before gelation and network architecture, which are related to bulk chemical formulation and reactivity. ${ }^{[2,3]}$ Understanding cure advancement has been the driving force for establishing process parameters for resins that undergo both cure and flow. The kinetics of conversion for reactive resins and their dependence on an activation energy has often been evaluated by curing at different temperatures and establishing the activation energy ${ }^{[4-8]} \mathrm{A}$ recent review related conversion to the capacity for molecular diffusion for several common polymerization 
schemes. ${ }^{[9]}$ Several theoretical and empirical viscosity advancement models were also summarized by Halley and Mackay. ${ }^{[10]}$ Recent publications have gauged both reactive stability and defined flow and processing characteristics for reactive injection molding resins and other flowing resins. ${ }^{[11-13]}$

Wong et al. have targeted research tied to a range of microelectronics problems, including flip-chip assemblies and underfill, by studying different isothermal temperature gelation processes and the resulting network structure of epoxy resins, which are closely related to the reliability of those assemblies. ${ }^{[14,15]}$ This was expanded on in a review published by $\mathrm{Li}$ and Wong. ${ }^{[16]}$

There has been common interest in the link between the viscosity and reaction of reactive dispersions ${ }^{[17-18]}$ and highly-crosslinkable photocurable polymers, in which rapid viscosity changes are induced. ${ }^{[18-19]}$ The allure of the power-law model, shown in Equation (1), is the relatively simple analysis of reaction kinetics that can ensue.

$$
\log \eta(t)=\log \eta_{0}+n t
$$

For the simplest power-law model, $\eta(t)$ is the timedependent viscosity, $\eta_{0}$ is the pre-gel initial viscosity at which crosslinking is initiated, $n$ is related to the rapidity of the viscosity rise, and $t$ is time. We analyzed both published data on curing epoxy resins including filled and unfilled resins, and photopolymerized resins where the kinetics of cure are more rapid. ${ }^{[19,20]}$

This power-law rheological model, while conceptually simple, can deviate significantly from observations as the network density rises. We considered whether other nonlinear analytical models could more accurately follow the dynamic viscosity changes induced by crosslinking. ${ }^{\text {[21-24] }}$ Others have used a Boltzmann sigmoidal model to describe other viscous fluids - including starches, ${ }^{[25]}$ siloxane-based impression compounds ${ }^{[23]}$ and other gels - undergoing a setting reaction. In our adapted model, we fitted the following Boltzmann sigmoidal function:

$$
\log \eta(t)=\log \eta_{\infty}+\frac{\log \left(\eta_{0}\right)-\log \left(\eta_{\infty}\right)}{\left(1+e^{\frac{t-t_{0}}{\Delta t}}\right)}
$$

where $\eta_{0}$ and $\eta_{\infty}$ are, respectively, the viscosities before initiating cure and after network formation at long times of transient cure. The induction time, $t_{0}$, corresponded to the time necessary a change viscosity from the initial viscosity $\log \eta_{0}$ to $\left(\log \eta_{0}+\log \eta\right) / 2$, and $\Delta t$ corresponds with the period associated with the sigmoidal transition region as viscosity deviates from $\eta_{0}$ in the semi-log linear regime. These parameters all vary with illumination intensity, temperature, oxygen content and other factors that regulate the kinetics of polymerization.

\section{Experimental Part}

Earlier published experiments used crosslinking mixtures of glycidyl end-capped poly[(bisphenol A)-co-epichlorohydrin] and glutaric acid (Aldrich Chemical Company, Inc.) combined with a Novalac epoxy resin (LBR-6) and 1-cyanoethyl-2-undecyl-1Himozolium trimellitate (C11Z-CNS) as model material for testing. ${ }^{[26]}$ The chemorheology experiments were originally carried out in a TA Instruments AR1000 rheometer using a parallel plate geometry stress rheometer at fixed curing temperatures $(150,160$, 170 and $180^{\circ} \mathrm{C}$ ) to simulate the heating profile conducted in underfilling.

Datasets from the published rheology experiments ${ }^{[26]}$ were subsequently inputted into Microcal Origin ${ }^{\mathrm{TM}}$, which has a 4parameter sigmoidal plot function included. We analyzed these curves with no constraints on the initial and terminal viscosity. Subsequent re-analyses fixed the initial viscosity, which led to a sigmoidal model with only three parameters.

\section{Results}

Comparisons of viscosity as a function of time and cure temperature are made between the original modified power-law analyses, ${ }^{[26]}$ shown in Equation (3), and the new three-parameter sigmoidal analysis in Equation (2); these are plotted in Figure 2 using the sigmoidal plot function, fixing only $\log \eta_{0}=-2.2 \mathrm{~Pa} \cdot \mathrm{s}$. One important feature of the original model was the linkage of the Arrhenius kinetics of reaction into the empirical chemorheological relation, resulting in Equation (3) and plotted in Figure 1. A second feature was that the original analysis was focused primarily on the lower viscosity regime, with $\eta<10 \mathrm{~Pa} \cdot \mathrm{s}$. The sigmoidal plot [from Equation (2)]

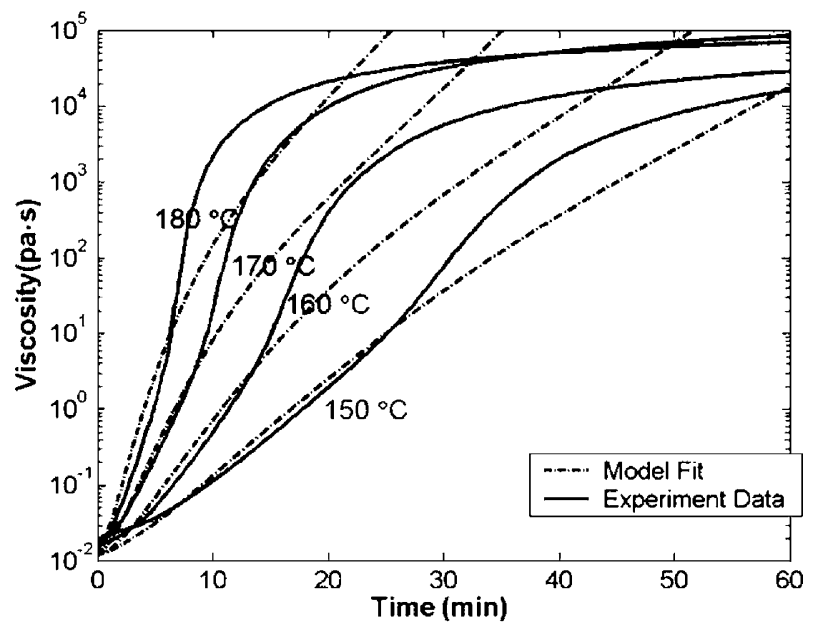

Figure 1. Plot representing the experimental data of the original manuscript and the model fit. [26] 


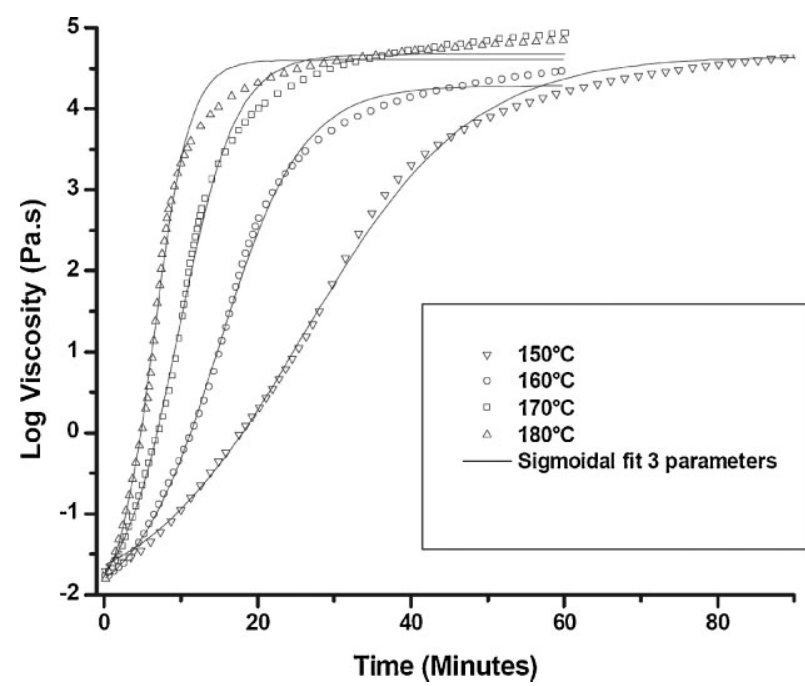

Figure 2. Sigmoidal re-analysis showing dynamic viscosity, as well as sigmoidal fit.

represents both the initial, low viscosity region as well as the asymptotic plateau of dynamic viscosity, where the rate of polymerization declines due to viscous constraints on the reactive end-groups. The experimental data matched the sigmoidal model up to a threshold viscosity of about $1 \times 10^{4} \mathrm{~Pa} \cdot \mathrm{s}$.

$$
\ln \eta=-42.54+3.27 \ln \bar{M}_{\mathrm{w}}+8608 \frac{1}{T}
$$

The three parameters modeled here as part of the sigmoidal analysis are shown, as a function of cure temperature, in Table 1. The expected trend of shorter induction times with increasing temperature, as well as a shorter period associated with the rate of viscosity rise was observed. The trend in the sigmoidal time constants plotted as a function of $1 / T$ is included in Figure 3 . Obviously, the reaction rate, regulated by an activation energy, is also affecting the viscous response, as it did with the power-law model; however, the time constants are not linear functions with a single activation energy. The

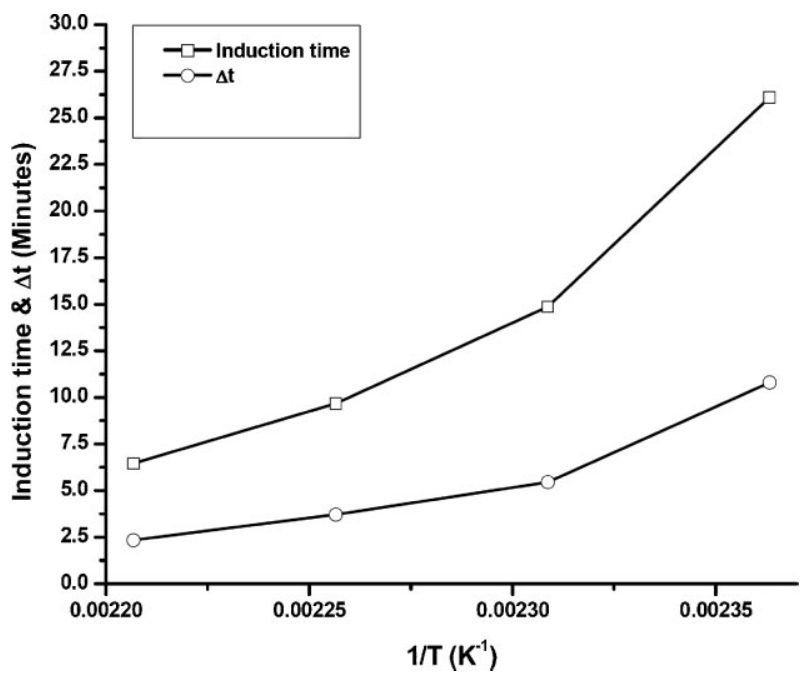

Figure 3. Kinetic time constants, $t_{0}$ and $\Delta t$, as a function of $1 / T$.

representation of $\Delta t$, which is inversely proportional to the rate of viscosity rise, is capturing a rate effect later in the conversion of the viscosity than the power-law model.

One legitimate question is whether more or fewer constraints on the 3 parameter model are appropriate. Fixing the terminal viscosity would yield only the two kinetic time constants, but this does not seem to capture the terminal network density also likely being a function of curing temperature. Since the resin formulation was not changed during these experiments, a fixed constraint on the initial viscosity seems reasonable. The cure temperature could have its own effect on the resin viscosity initially, but this seems to be a small variation, as seen in Figure 1.

\section{Conclusion}

Dynamic viscosity measurements of chip-underfill material were re-modeled using a Boltzmann sigmoidal model. The re-analysis, while emipirical, yields several time

Table 1. Tabulated parameters for the Boltzmann sigmoidal analysis for the epoxy resin.

\begin{tabular}{|c|c|c|c|c|c|}
\hline \multirow[t]{2}{*}{$\begin{array}{l}\text { Curing } \\
\text { temperature }\end{array}$} & $\begin{array}{c}\log \text { (initial viscosity) } \\
\text { (fixed) }\end{array}$ & $\begin{array}{c}\log \\
\text { (final viscosity) }\end{array}$ & $\begin{array}{l}\text { Induction } \\
\text { time }\left(t_{0}\right)\end{array}$ & $\Delta t$ & $\begin{array}{c}\text { Correlation } \\
\text { coefficient }\left(R^{2}\right)\end{array}$ \\
\hline & $\mathrm{Pa} \cdot \mathrm{s}$ & $\mathrm{Pa} \cdot \mathrm{s}$ & $\min$ & $\min$ & \\
\hline 150 & -2.2 & 4.66 & 26.1 & 10.79 & 0.998 \\
\hline 160 & -2.2 & 4.29 & 14.86 & 5.45 & 0.998 \\
\hline 170 & -2.2 & 4.67 & 9.68 & 3.71 & 0.996 \\
\hline 180 & -2.2 & 4.6 & 6.46 & 2.34 & 0.994 \\
\hline
\end{tabular}


constants that have physical significance in the interpretation of reaction kinetics. The sigmoidal model describes a wider range of dynamic viscosity for candidate underfill resins than the more common power-law models, which have been adapted to include the kinetics of reaction. The determination of a single Arrhenius-type rate constant was not evident from the dynamic viscosity sigmoidal analysis, but further refinement might more strongly integrate reaction kinetics into the sigmoidal model.

Received: June 9, 2008; Accepted: August 4, 2008; DOI: 10.1002/ mame.200800170

Keywords: chemorheology; crosslinking; epoxy; rheology; thermosets

[1] S. A. Madbouly, T. Ougizawa, J. Macromol. Sci. -Phys. 2004, B43, 655 .

[2] B. Gaur, J. S. P. Rai, Polym. -Plast. Technol. Eng. 2006, 45, 197.

[3] H. T. Chiu, P. A. Tsai, T. C. Cheng, J. Mater. Eng. Perform. 2006, 15,81

[4] Y. S. Yang, L. Suspene, Polym. Eng. Sci. 1991, 31, 321.

[5] J. M. Laza, J. L. Vilas, M. Rodriguez, M. T. Garay, F. Mijangos, L. M. Leon, J. Appl. Polym. Sci. 2002, 83, 57.

[6] J. M. Laza, J. L. Vilas, F. Mijangos, M. Rodriguez, L. M. Leon, J. Appl. Polym. Sci. 2005, 98, 818.
[7] M. E. Ryan, A. Dutta, Polymer 1979, 20, 203.

[8] A. Y. Malkin, Polym. Eng. Sci. 1980, 20, 1035.

[9] D. S. Achilias, Macromol. Theory Simul. 2007 16, 319.

[10] P. J. Halley, M. E. Mackay, Polym. Eng. Sci. 1996, 36, 593.

[11] T. A. Bullions, J. E. McGrath, A. C. Loos, Polym. Eng. Sci. 2002, 42, 2182.

[12] A. N. Derose, M. Yuan, T. A. Osswald, J. M. Castro, Polym. -Plast Technol. Eng. 2002, 41, 383.

[13] K. C. Cheng, W. Y. Chiu, K. H. Hsieh, C. C. M. Ma, J. Mater. Sci. 1994, 29, 721.

[14] Z. Zhang, E. Beatty, C. P. Wong, Macromol. Chem. Phys. 2005 206, 869.

[15] Z. Zhang, T. Yamashita, C. P. Wong, Macromol. Mater. Eng. 2003, 288, 365.

[16] Y. Li, C. P. Wong, Mater. Sci. Eng. 2006, 51, 1.

[17] P. I. Dolez, A. Gf, B. J. Love, Separation Sci. Technol. 2002, 37 2007.

[18] B. J. Love, Particulate Sci. Technol. 2004, 22, 285.

[19] F. Piguet-Ruinet, B. J. Love, J. Appl. Polym. Sci. 2008, 107, 1523.

[20] B. J. Love, F. Piguet-Ruinet, J. Appl. Polym. Sci. 2007, 106, 3605.

[21] K. J. Kim, B. K. Kim, J. Appl. Polym. Sci. 1993, 48, 981.

[22] M. Uhniat, M. Zemlak, J. John, R. Szulc, Polimery 2003, 48, 709.

[23] J. C. Berg, G. H. Johnson, X. Lepe, S. Adan-Plaza, J. Prosthetic Dentistry 2003, 90, 150.

[24] B. J. Love, F. Piguet-Ruinet, F. Teyssier, J. Polym. Sci., Part B: Polym. Phys. 2008, in press.

[25] K. Israkarn, P. Hongsprabhas, P. Hongsprabhas, Carbohydrate Polym. 2007, 68, 314.

[26] Y. Y. Sun, Z. Zhang, C. P. Wong, Macromol. Mater. Eng. 2005 , 290, 1204. 